\title{
A clinico-genomic analysis of soft tissue sarcoma patients reveals CDKN2A deletion as a biomarker for poor prognosis
}

\author{
Nam Q. Bui ${ }^{1 *} \mathbb{D}$, Joanna Przybyl², Sally E. Trabucco ${ }^{3}$, Garrett Frampton ${ }^{3}$, Trevor Hastie ${ }^{4}$, Matt van de Rijn ${ }^{2}$ \\ and Kristen N. Ganjoo'
}

\begin{abstract}
Background: Sarcomas are a rare, heterogeneous group of tumors with variable tendencies for aggressive behavior. Molecular markers for prognosis are needed to risk stratify patients and identify those who might benefit from more intensive therapeutic strategies.

Patients and methods: We analyzed somatic tumor genomic profiles and clinical outcomes of 152 soft tissue (STS) and bone sarcoma (BS) patients sequenced at Stanford Cancer Institute as well as 206 STS patients from The Cancer Genome Atlas. Genomic profiles of 7733 STS from the Foundation Medicine database were used to assess the frequency of CDKN2A alterations in histological subtypes of sarcoma.

Results: Compared to all other tumor types, sarcomas were found to carry the highest relative percentage of gene amplifications/deletions/fusions and the lowest average mutation count. The most commonly altered genes in STS were TP53 (47\%), CDKN2A (22\%), RB1 (22\%), NF1 (11\%), and ATRX (11\%). When all genomic alterations were tested for prognostic significance in the specific Stanford cohort of localized STS, only CDKN2A alterations correlated significantly with prognosis, with a hazard ratio (HR) of 2.83 for overall survival $(p=0.017)$. These findings were validated in the TCGA dataset where CDKN2A altered patients had significantly worse overall survival with a HR of $2.7(p=0.002)$. Analysis of 7733 STS patients from Foundation One showed high prevalence of CDKN2A alterations in malignant peripheral nerve sheath tumors, myxofibrosarcomas, and undifferentiated pleomorphic sarcomas.
\end{abstract}

Conclusion: Our clinico-genomic profiling of STS shows that CDKN2A deletion was the most prevalent DNA copy number aberration and was associated with poor prognosis.

Keywords: Soft tissue sarcoma, Genomics, Prognostic markers, CDKN2A

\section{Background}

Sarcomas are rare, heterogeneous mesenchymal tumors with variable tendencies for aggressive behavior. The most important clinical risk factors for recurrence are tumor size, grade, and histology [1] with high risk tumors being classified as size $>5 \mathrm{~cm}$, FNCLCC grade II-III, and an aggressive histology (ex. undifferentiated pleomorphic sarcoma, dedifferentiated liposarcoma, leiomyosarcoma,

\footnotetext{
*Correspondence: nambui@stanford.edu

${ }^{1}$ Department of Medicine (Oncology), Stanford University School

of Medicine, 875 Blake Wilbur Drive, Stanford, CA 94305, USA

Full list of author information is available at the end of the article
}

etc.). However, even among high risk localized sarcomas, there are considerable differences in clinical outcomes with approximately half of patients achieving a long term remission, while half relapse within 5 years [2]. Attempts to decrease the rate of disease recurrence with adjuvant chemotherapy demonstrated either negative $[3,4]$ or marginally positive results $[2,5]$. New prognostic stratification markers are needed to help identify patients at risk of recurrence and possibly apply more intensive or novel treatments in this cohort.

Cancer genomics is playing an increasingly vital role in prognostic stratification of cancer patients. Prominent examples include karyotype analysis in leukemia 
[6], RNA transcriptome analysis in breast cancer [7], DNA methylation analysis in glioblastoma [8], and DNA mutations in head and neck cancer [9]. Next generation sequencing has entered into mainstream clinical practice with increasing adoption for metastatic cancer patients and recent FDA approval and subsequent Medicare coverage of a sequencing companion diagnostic [10]. With the proliferation of sequencing information, there is opportunity to discover new prognostic correlations from mutational data, especially for rare tumors with limited prognostic features.

In this study, we examined 152 sarcoma patients treated at Stanford Cancer Institute by tumor genome sequencing and explored associations between genomic alterations and outcomes. We also analyzed data from The Cancer Genome Atlas (TCGA) sarcoma project, to independently validate findings from the Stanford cohort. In addition, we explored the Foundation Medicine sequencing database to describe the landscape of CDKN2A alterations in STS.

\section{Methods \\ Patient Selection}

Between 2012 and 2017, 1291 patients at Stanford Cancer Institute had tumor genomic sequencing performed with hybrid capture based next generation sequencing (Foundation Medicine, Cambridge, MA) [11, 12]. One hundred fifty-two of these patients had soft tissue or bone sarcoma. Patient data including demographics and clinical data were abstracted retrospectively from the medical chart. Data cut-off was December 19, 2018. This study was approved by the Stanford University institutional review board (IRB).

TCGA DNA point mutation and copy number data from 206 sarcoma patients were analyzed through cBioPortal (accessed on 02/19/2019) [13]. In addition, we analyzed the CDKN2A mutation status including DNA copy number changes, genomic rearrangements, and SNVs (somatic and germline [14]) in 7733 soft tissue sarcoma patients analyzed by Foundation Medicine. These samples were sequenced as part of routine clinical care following previously described methods $[11,12]$. Samples were submitted to a CLIA-certified, New York State-accredited, and CAP-accredited laboratory (Foundation Medicine) for hybrid capture followed by nextgeneration sequencing using the FoundationOneHeme ${ }^{\circledR}$, FoundationOne $^{\circledR}$, or FoundationOneCDx ${ }^{\circledR}$ platforms.

\section{Statistical analysis}

The Cox proportional hazards model was used for comparative survival analysis. Overall survival was defined as time from diagnosis until patient death. Time to recurrence was defined as time from diagnosis until local or distant recurrence. Time to treatment failure was defined as time from the first day of therapy until radiographic/ clinical progression or death. $\mathrm{p}$ values $<0.05$ were considered significant. In the TCGA survival analysis, associated risk factors for prognosis were determined by running multiple univariate Cox proportional hazards models for age, FNCLCC grade, stage, tumor size, histology, and $C D K N 2 A$ status. Of those, only age, stage, tumor size, and $C D K N 2 A$ status significantly affected prognosis and were thus incorporated into the final multivariate Cox model. Tumor size violated the proportional hazards assumption and therefore a time-transforming function (tt) was used. Statistical analysis was performed in R (version 3.3.2) [15] with the survival package (Version 2.41.3) [16].

\section{Results}

\section{Patient characteristics}

Characteristics of 152 sarcoma patients treated at the Stanford Cancer Institute are summarized in Table 1. The average age of the patients was 54.5, ranging from 15 to 90 years old. The vast majority of tumors were soft tissue sarcoma $(n=134)$ with a small number of primary bone sarcomas $(n=12)$. The most common tumor type was leiomyosarcoma (LMS) $(n=25)$, followed by undifferentiated pleomorphic sarcoma (UPS) $(n=23)$, myxofibrosarcoma (MFS) $(n=13)$, liposarcoma (LPS) $(n=13: 11$ dedifferentiated LPS, 1 myxoid LPS, 1 pleomorphic LPS), and malignant peripheral nerve sheath tumor (MPNST) $(\mathrm{n}=9)$. Most patients had localized sarcoma at diagnosis $(70 \%, n=106)$, although almost all patients were diagnosed with or developed metastases during the course of their disease $(89 \%, \mathrm{n}=136)$. This high rate of metastasis was most likely due to selection bias as genomic sequencing was almost only performed for potential therapeutic options in refractory patients. The extremity was the most common site of disease $(30 \%, n=46)$, followed by pelvic $(20 \%, n=31)$, and trunk $(10 \%, n=15)$. Adjuvant chemotherapy was administered to $41 \%(\mathrm{n}=48)$ of patients and adjuvant radiotherapy was given to $66 \%$ $(n=78)$ of patients.

\section{Soft tissue and bone sarcoma are defined by copy number changes and fusion events}

When compared to other tumor types, the predominant genomic aberrations in soft tissue and bone sarcomas were DNA copy number and chromosomal translocations (Fig. 1a). Based on the cohort of patients treated at Stanford Cancer Institute, sarcomas had the lowest average number of single nucleotide variants (SNVs), averaging 1.7 SNVs per tumor compared to 6.1 SNVs per tumor in melanoma, which had the highest mutation rate among the analyzed tumor types. Conversely, 
Table 1 Demographics of patients treated at Stanford Cancer Institute and TCGA

\begin{tabular}{|c|c|c|}
\hline Characteristic & $\begin{array}{l}\text { Stanford } \\
\text { patients } \\
(n=152)\end{array}$ & TCGA $(n=206)$ \\
\hline Age at diagnosis & $54.5(15-90)$ & $60(20-90)$ \\
\hline \multicolumn{3}{|l|}{ Sex } \\
\hline Male & $74(48.7 \%)$ & $94(46 \%)$ \\
\hline Female & $78(51.3 \%)$ & $112(54 \%)$ \\
\hline \multicolumn{3}{|l|}{ Race or ethnic group } \\
\hline Caucasian & $104(68 \%)$ & N/A \\
\hline Hispanic & $22(14 \%)$ & \\
\hline Asian & $22(14 \%)$ & \\
\hline Other & $4(3 \%)$ & \\
\hline \multicolumn{3}{|l|}{ Tumor histology } \\
\hline Leiomyosarcoma & 25 & 80 \\
\hline $\begin{array}{l}\text { Undifferentiated pleomorphic } \\
\text { sarcoma }\end{array}$ & 23 & 44 \\
\hline Sarcoma (NOS) & 14 & \\
\hline Myxofibrosarcoma & 13 & 17 \\
\hline Liposarcoma & 13 & 50 \\
\hline $\begin{array}{l}\text { Malignant peripheral nerve sheath } \\
\text { tumor }\end{array}$ & 9 & 5 \\
\hline Synovial sarcoma & 7 & 10 \\
\hline Osteosarcoma & 5 & \\
\hline Angiosarcoma & 5 & \\
\hline Rhabdomyosarcoma & 3 & \\
\hline Ewing's sarcoma & 3 & \\
\hline Chondrosarcoma & 3 & \\
\hline Malignant phyllodes tumor & 3 & \\
\hline \multicolumn{3}{|l|}{ Local or metastatic } \\
\hline Local & $106(70 \%)$ & $89(43 \%)$ \\
\hline Locally advanced & $8(5 \%)$ & \\
\hline Metastatic & $38(25 \%)$ & $46(22 \%)$ \\
\hline Unknown & & $71(34 \%)$ \\
\hline \multicolumn{3}{|l|}{ Site } \\
\hline Extremity & $46(30 \%)$ & $62(30 \%)$ \\
\hline Pelvic & $31(20 \%)$ & $33(16 \%)$ \\
\hline Trunk & $15(10 \%)$ & $9(4 \%)$ \\
\hline Retroperitoneum & $21(14 \%)$ & $87(42 \%)$ \\
\hline Spine & $9(6 \%)$ & 0 \\
\hline Breast & $6(4 \%)$ & 0 \\
\hline Lungs & $4(3 \%)$ & $2(1 \%)$ \\
\hline Other & $10(7 \%)$ & $13(6 \%)$ \\
\hline Size $(\mathrm{cm})$ & $11.4(2-42)$ & $12.7(1.2-39.5)$ \\
\hline \multicolumn{3}{|l|}{ FNCLCC grade } \\
\hline I & $7(5 \%)$ & $14(7 \%)$ \\
\hline$\|$ & $23(15 \%)$ & $112(54 \%)$ \\
\hline III & $37(24 \%)$ & 80 (39\%) \\
\hline N/A & 85 (56\%) & \\
\hline
\end{tabular}

Table 1 (continued)

\begin{tabular}{lll}
\hline Characteristic & $\begin{array}{l}\text { Stanford } \\
\text { patients } \\
(\mathbf{n = 1 5 2})\end{array}$ & TCGA (n=206) \\
\hline $\begin{array}{l}\text { Adjuvant chemotherapy } \\
\text { Yes }\end{array}$ & \\
No & $48(41 \%)$ & $47(23 \%)$ \\
N/A & $70(59 \%)$ & $150(73 \%)$ \\
Adjuvant radiotherapy & & $9(4 \%)$ \\
Yes & & \\
No & $78(66 \%)$ & $58(28 \%)$ \\
N/A & $37(31 \%)$ & $139(67 \%)$ \\
\hline
\end{tabular}

sarcomas had relatively high percentage of copy number and fusion events, representing 57\% of all gene alterations compared to $7 \%$ for renal neoplasms, which was the tumor type with the lowest frequency of copy number alterations. The number of "targetable" mutations that led to non-standard of care treatment options was low, with only 8 patients receiving targeted therapy (5\%). Of these 8 patients, one patient had a complete response (myopericytoma with NTRK fusion on larotrectinib study), one patient had stable disease for 9 months (osteosarcoma with NF2 mutation on everolimus), two patients discontinued treatment due to drug toxicity, and 4 patients progressed rapidly on first follow up scan. The frequency and distribution of genomic alterations across different tumor types is summarized in Fig. 1b. The most frequently affected gene was TP53 (47\%), followed by CDKN2A (22\%), RB1 (22\%), NF1 (11\%), and ATRX (11\%). The majority of alterations in TP53, NF1, and ATRX were point mutations while the predominant alterations in $C D K N 2 A$ and $R B 1$ were copy number losses. Other than the pathognomonic $M D M 2$ amplification in liposarcoma, there were no altered genes that clustered into specific histologic types (Fig. 1b, top colored bar). Fusion driven sarcomas (Fig. 1b, purple bar), did not tend to have many co-occurring mutations.

\section{CDKN2A aberrations are associated with poor prognosis in soft tissue sarcoma}

Next, we sought to determine whether the most frequent genomic alterations in sarcoma patients treated at Stanford Cancer Institute correlated with prognosis. In order to standardize the patient population, we selected only patients with STS (excluding GISTs and primary bone sarcomas) and localized disease at diagnosis $(n=96)$. Of note there were 2 patients with spindle cell histologies 
a

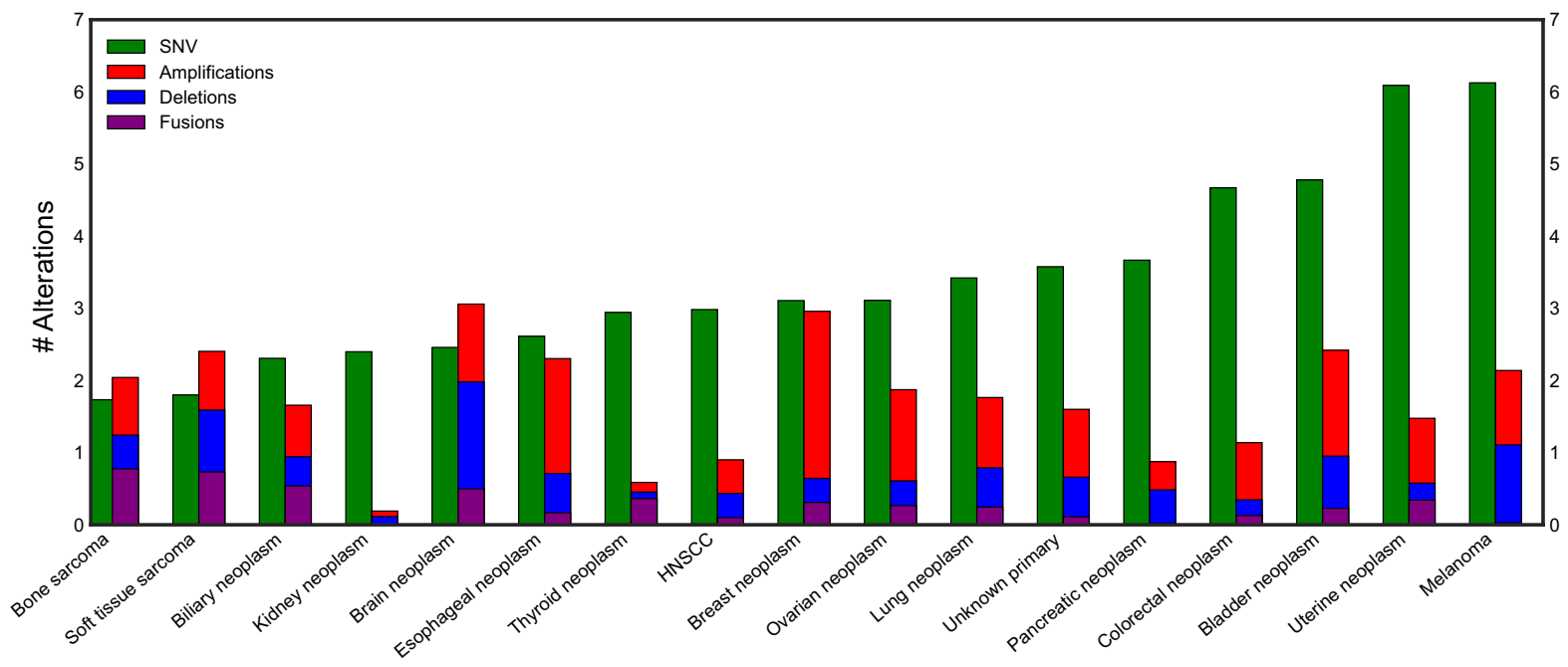

b

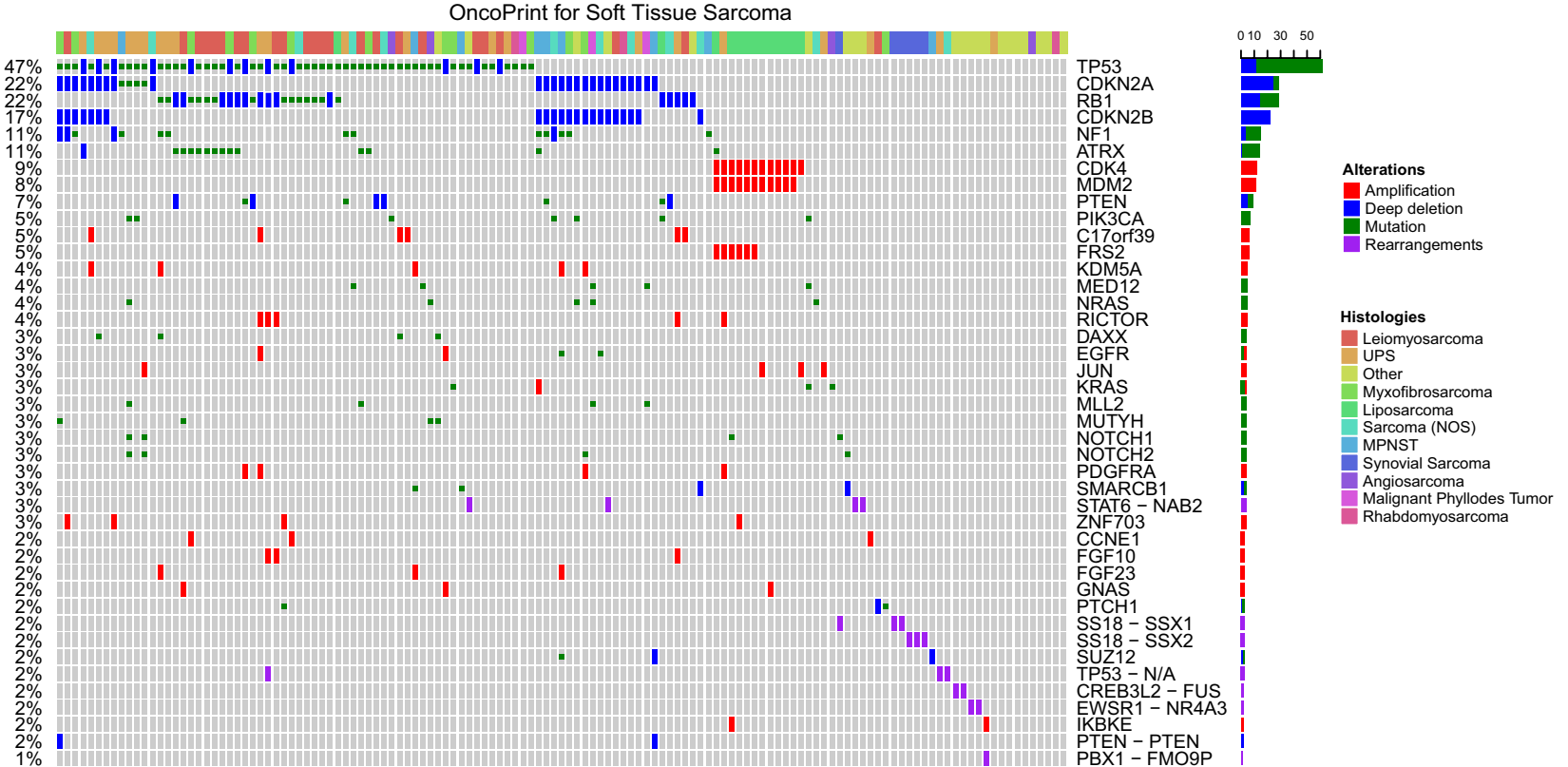

Fig. 1 Genomic landscape of bone and soft tissue sarcoma. a Average frequency per patient of SNVs, amplifications, deletions, and fusions per cancer type for 1291 Stanford patients with tumor sequencing. When ordered by average number of SNVs, bone and soft tissue have the lowest amount of SNVs while having the highest relative proportion of copy number and structural rearrangements. b Oncoprint [30] plot of genomic alterations in soft tissue sarcoma clustered by frequency. The genes are ordered from most frequent (top) to least frequent (bottom). Tumor histology is shown on the topmost colored bar

(See figure on next page.)

Fig. 2 Clinical outcomes for localized soft tissue sarcomas with genomic sequencing treated at Stanford $(n=96)$. a Forest plot of most commonly altered 8 genes with prognosis reveals only CDKN2A to be significantly associated with worse prognosis. $\mathbf{b}$ Kaplan-Meier plot of overall survival for CDKN2A altered vs. non-CDKN2A altered patients $(p=0.017)$. $\mathbf{c}$ Histologic distribution of all patients $(\mathbf{d})$ and CDKN2A altered patients (C2) reveals increases in representation of MPNST, MFS, and UPS with decreases in LMS and LPS. e Kaplan-Meier plot of time to recurrence for CDKN2A altered vs. non-CDKN2A altered patients $(p=0.09)$. $\mathbf{f}$ Kaplan-Meier plot of time to treatment failure for first line systemic chemotherapy for CDKN2A altered vs. non-CDKN2A altered patients $(p=0.29)$ 
a

Gene $n$ HR

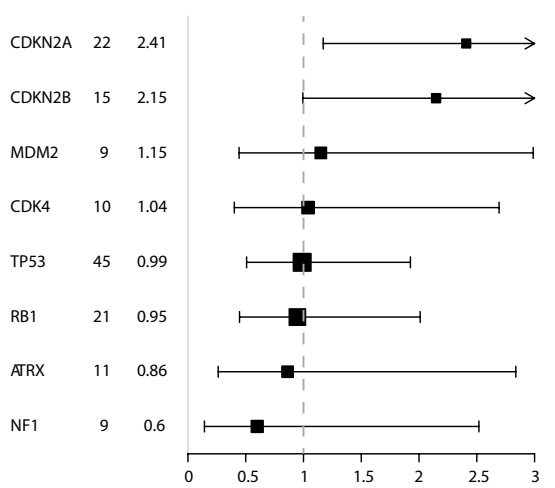

c

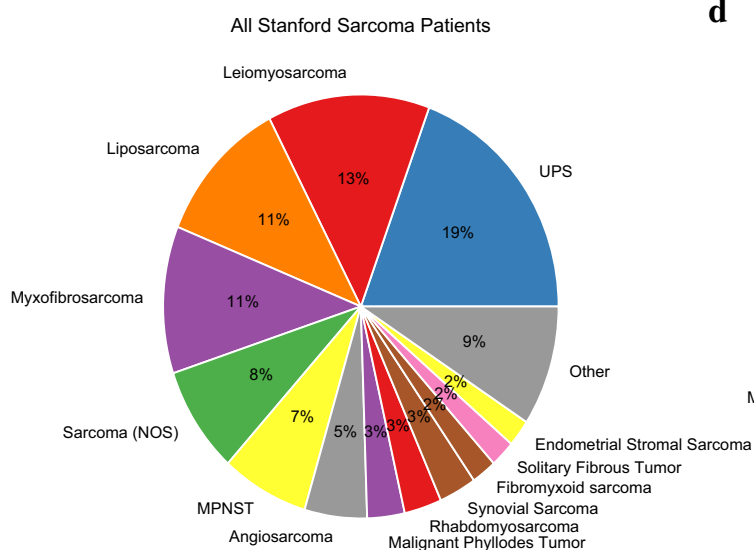

Angiosarcoma Malignant Phyllodes Tumor

e

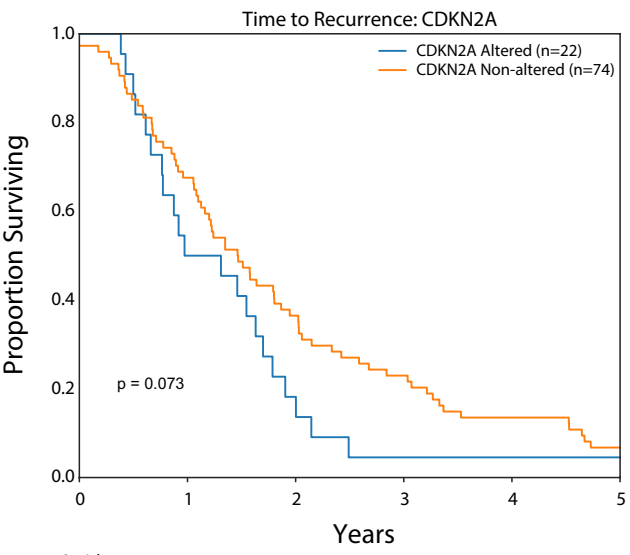

At risk
CDKN2A Altered $(\mathrm{n}=22)$
22 CDKN2A Non-altered $(n=74) 74$ b

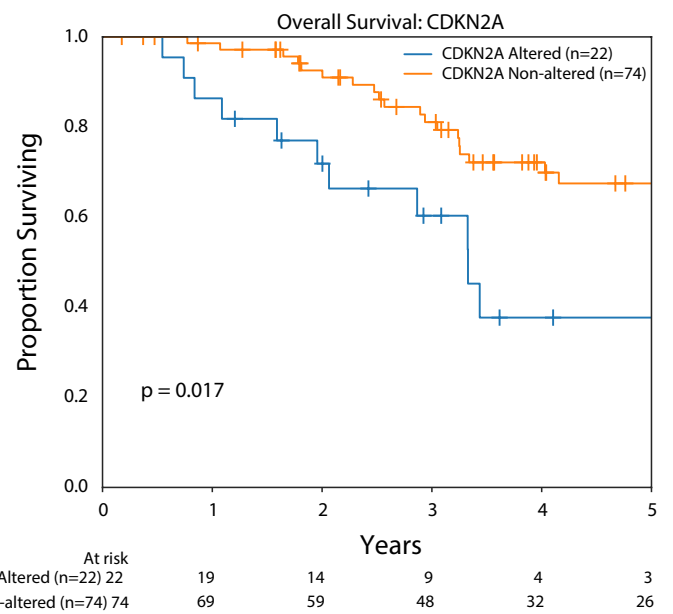

d

CDKN2A Sarcoma Patients

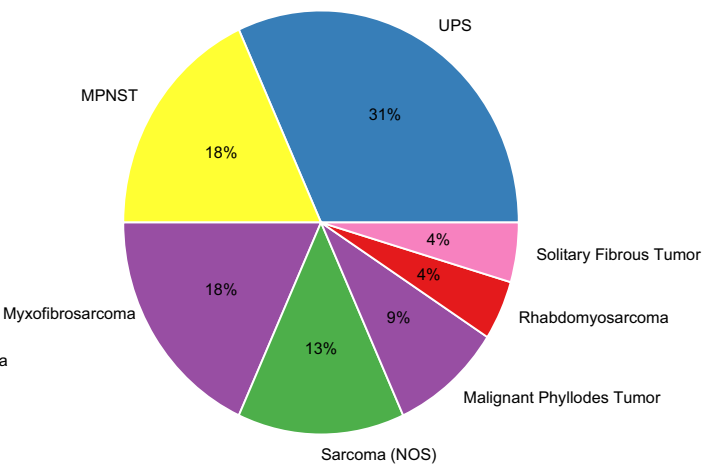

f

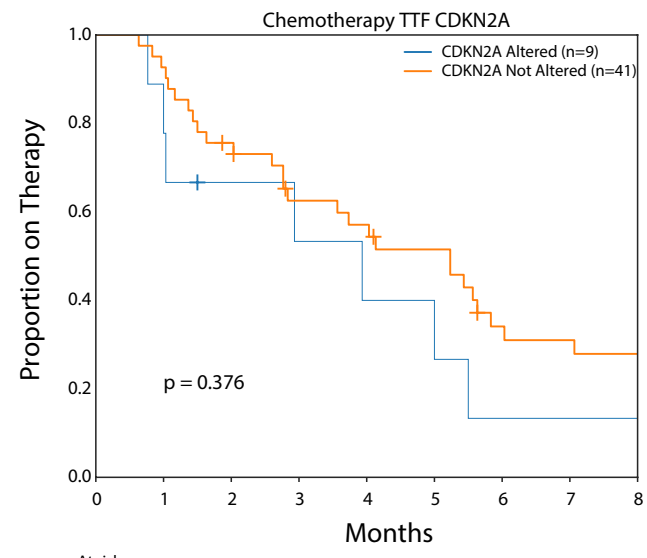

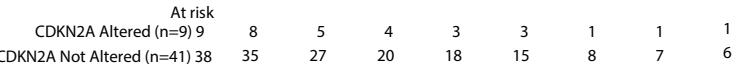


originating in bone that were included as they were treated under soft tissue sarcoma protocols. In a Cox proportional hazards model, adjusted for age, only CDKN2A alterations were associated with an effect on prognosis with a significantly worse overall survival (OS) (HR 2.83, mOS 3.3 vs. 7.7 years, $\mathrm{p}=0.017$, Fig. $2 \mathrm{a}, \mathrm{b})$. No other genetic alteration was found to significantly associate with survival. Twenty-two patients (23\%) had CDKN2A alterations of which $18(82 \%)$ were homozygous deletions, $3(14 \%)$ were nonsense mutations, and $1(5 \%)$ was a loss of function SNV. Histological distributions of all sarcoma patients (Fig. 2c) compared with patients with aberrant CDKN2A (Fig. 2d), showed an increase in the representation of UPS, MPNST, and MFS patients, and a decrease in the amount of LMS and LPS patients in those with $C D K N 2 A$ loss. Analysis of time to recurrence (either local or metastatic) showed that there was a trend to earlier recurrence with $C D K N 2 A$ altered patients (median 0.87 vs. 1.2 years, logrank $\mathrm{p}=0.073$, Fig. $2 \mathrm{e}$ ), however, this was not statistically significant. There was no difference in time to treatment failure (TTF) for first line chemotherapy, with a median time to progression of 3.9 vs. 5.2 months $(\mathrm{p}=0.38)$ for $C D K N 2 A$ altered vs. non-altered patients (Fig. 2f). These results suggest that suggests that there may be an inherently more aggressive biology in patients with alterations in the CDKN2A gene, with an earlier time to recurrence from initial surgery, but no substantial increase in resistance to chemotherapy once disease becomes metastatic.

We validated these findings in the TCGA sarcoma dataset, which contains DNA copy number and mutation data from 206 primary untreated STS patients and clinical follow up information. The dataset focuses on 6 major sarcoma subtypes including dedifferentiated LPS (DDLPS), LMS, UPS, MFS, MPNST, and synovial sarcoma (SS). There were 25 tumors with CDKN2A homozygous deletion, 180 were wild type, and 1 case had CDKN2A amplification (TCGA PanCancer Atlas data, accessed on 2/19/2019 through cBioPortal). There was only one patient who had a $C D K N 2 A$ nonsense mutation and this patient also had concurrent $C D K N 2 A$ copy number loss. Among tumors with CDKN2A loss there was an increased representation of MPNST, MFS, and UPS patients and a decreased ratio of LMS and LPS, similar to our Stanford data set (Fig. 3c, d). In a multivariate Cox proportional hazards model adjusted for age, stage, and tumor size, $C D K N 2 A$ alterations were significantly associated with a worse prognosis (HR 2.7, mOS 2.5 vs. 6.7 years, $p=0.002$, Fig. $3 a$ ). Other significant risk factors influencing outcome were age and presence of metastases at diagnosis (Table 2). We also tested other potential risk factors such as tumor histology (Fig. $3 \mathrm{~b}$ ) and grade in univariate Cox models but neither were significantly correlated with survival outcomes. Exploratory analysis of $C D K N 2 A$-associated prognosis within each histology was performed, albeit at considerable loss of statistical power due to individually small sample size, and demonstrated pronounced survival differences in MFS, STLMS, UPS, and SS (Additional file 1: Figure S1).

\section{The landscape of CDKN2A genomic alterations in soft tissue sarcoma}

Sarcomas are extremely rare tumors with less than 15,000 new cases diagnosed each year in the United States [17]. Therefore, large, multi-institutional, central databases are critical for analysis of these rare tumors, as it is difficult for a single institution to accrue a sufficiently large patient cohort for comprehensive analysis. We queried the Foundation Medicine database that has sequencing data on 7733 soft tissue sarcomas from numerous institutions for alterations in $C D K N 2 A$. The frequency of genomic aberrations in $C D K N 2 A$ gene in this multi-institutional cohort was similar to our Stanford cohort, with an alteration rate of $16.7 \%$ (Table 3). Almost all of these were copy number changes (loss) at $14.1 \%$, with smaller numbers of SNVs (2.3\%), and gene rearrangements $(0.5 \%)$. There is emerging data on germline CDKN2A mutations that predispose towards the development of sarcoma [18], however, we found that these cases were exceedingly rare $(0.2 \%)$. When broken down into histologic type (Fig. 4), the most commonly affected tumor type was MPNST $(60.7 \%, \mathrm{n}=262)$, in which $C D K N 2 A$ loss has been shown previously to be a defining event for the malignant transformation of neurofibromas [19, 20]. Other commonly $C D K N 2 A$-mutated tumors were myxofibrosarcomas $(29.3 \%, \mathrm{n}=140)$, undifferentiated pleomorphic sarcomas $(29 \%, n=372)$, and fibrosarcomas $(26.3 \%, n=99)$. Leiomyosarcomas and liposarcomas were found to infrequently have $C D K N 2 A$ aberrations at $\mathrm{a} \leq 10 \%$ rate. These findings correlate with the histologic distributions both in the Stanford data set as well as the TCGA cohort (Additional file 1: Figure S2).

\section{Discussion}

In our single institution cohort, we show that $C D K N 2 A$ deletions were the most prevalent DNA copy number aberrations in STS and these aberrations were associated with poor clinical outcome. Based on an independent cohort of patients in the TCGA study, we validated $C D K N 2 A$ loss as an adverse prognostic factor. Finally, we illustrated the landscape of $C D K N 2 A$ loss across a large multi-institutional sequencing database of STS.

Several previous studies have proposed other molecular prognostic markers in sarcoma, however, they have not yet been introduced in the clinical practice. The recent TCGA analysis of STS [21] identified poor 


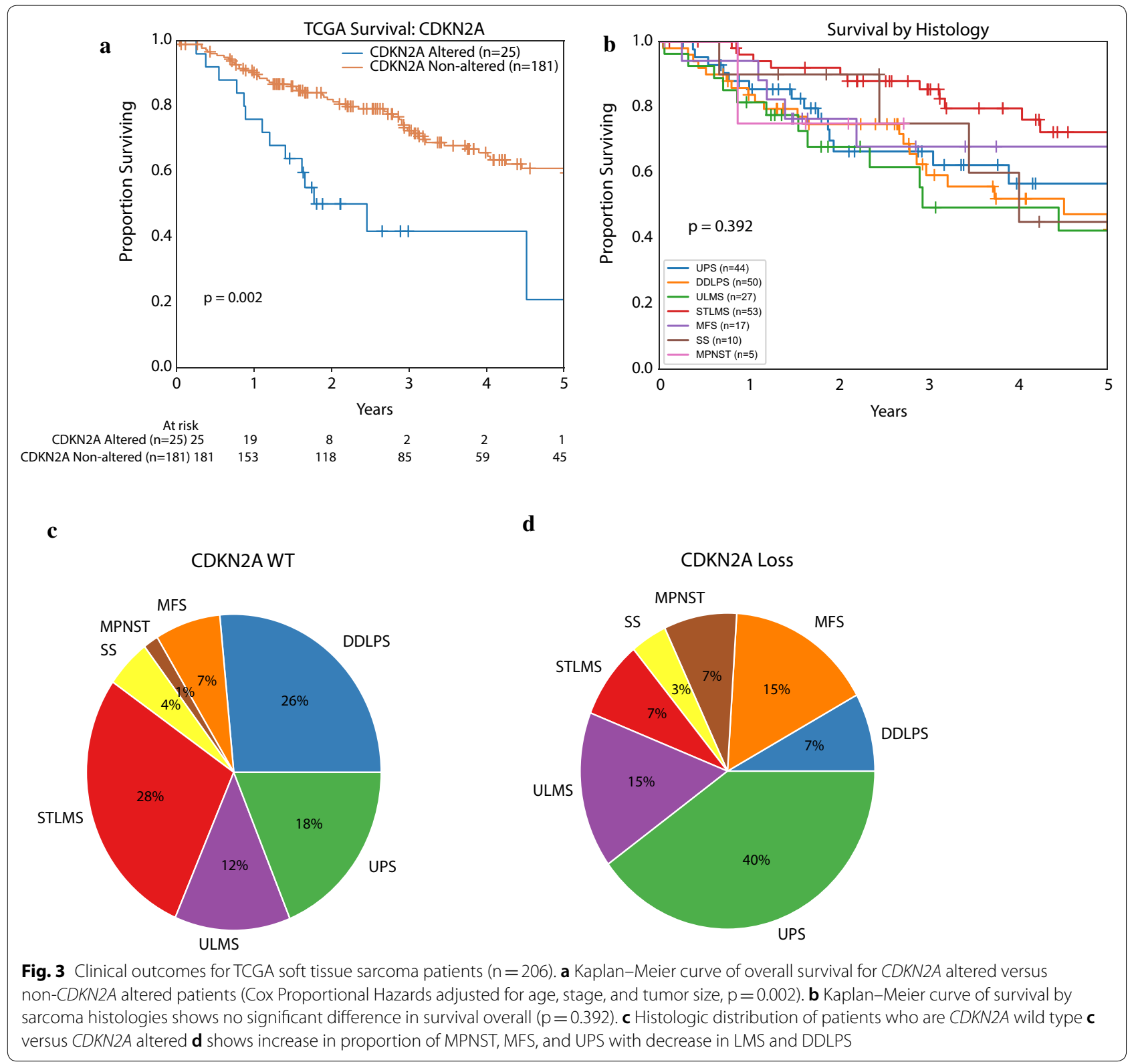

performing cohorts of patients within DDLPS and soft tissue LMS. The DDLPS patients with poor clinical outcome were characterized by hypermethylation and

Table 2 Cox proportional hazards model for TCGA patients

\begin{tabular}{lll}
\hline Characteristic & Hazard ratio $(\mathbf{9 5} \% \mathrm{Cl})$ & p value \\
\hline CDKN2A alteration & $2.7(1.4-5.1)$ & $0.002^{*}$ \\
Unknown stage (reference) & 1 & \\
Localized tumor at diagnosis & $0.42(0.23-0.79)$ & $0.007^{*}$ \\
Metastasis at diagnosis & $1.79(1.06-3)$ & $0.03^{*}$ \\
Age at diagnosis & $1.03(1.01-1.05)$ & $0.002^{*}$ \\
Tumor size $(\mathrm{cm})$ & $1(0.99-1.01)$ & 0.053 \\
\hline
\end{tabular}

certain chromosomal amplifications, while the LMS patients with adverse outcome were characterized by high expression levels of microRNA miR-181b-5p. Other high-throughput studies identified a CINSARC gene expression signature related to mitosis and chromosome integrity that identified high-risk patients and outperformed the histology-based grading system in sarcomas with complex genomic profiles such as LMS, UPS and DDLPS, GISTs, and synovial sarcoma [22, 23]. In regards to the prognostic impact of CDKN2A loss in sarcoma, previous work had suggested that its loss might have prognostic significance in Ewing's sarcoma [24-26], however, a large analysis of 568 Ewing's patients enrolled on 
Table 3 Foundation one histology distribution

\begin{tabular}{|c|c|c|c|c|c|c|c|c|c|}
\hline Disease & $\mathrm{n}$ & All CDKN2A & Copy \# & Rearrangements & SNV: somatic & SNV: germline & SNV: unknown & SNV: all & $\begin{array}{l}\text { Multiple } \\
\text { alterations }\end{array}$ \\
\hline All soft tissue sarcoma & 7733 & 16.7 & 14.0 & 0.4 & 1.1 & 0.2 & 0.7 & 2.0 & 0.3 \\
\hline $\begin{array}{l}\text { Malignant peripheral } \\
\text { nerve sheath tumor } \\
\text { (MPNST) }\end{array}$ & 262 & 60.7 & 48.1 & 5.3 & 5.0 & 0.4 & 1.5 & 6.9 & 0.4 \\
\hline Myxofibrosarcoma & 140 & 29.3 & 23.6 & 1.4 & 2.1 & 0.7 & 0.7 & 3.6 & 0.7 \\
\hline $\begin{array}{l}\text { Undifferentiated } \\
\text { pleomorphic sarcoma } \\
\text { (UPS) }\end{array}$ & 372 & 29 & 21.5 & 0.3 & 5.1 & 0.0 & 1.3 & 6.5 & 0.8 \\
\hline $\begin{array}{l}\text { Soft tissue sarcoma } \\
\text { (NOS) }\end{array}$ & 1476 & 28.9 & 25.4 & 0.4 & 1.3 & 0.3 & 1.1 & 2.6 & 0.5 \\
\hline Fibrosarcoma & 99 & 26.3 & 22.2 & 0.0 & 2.0 & 0.0 & 1.0 & 3.0 & 1.0 \\
\hline Clear cell sarcoma & 72 & 23.6 & 18.1 & 0.0 & 0.0 & 1.4 & 2.8 & 4.2 & 1.4 \\
\hline Epithelioid sarcoma & 72 & 20.8 & 18.1 & 0.0 & 2.8 & 0.0 & 0.0 & 2.8 & 0.0 \\
\hline $\begin{array}{l}\text { Perivascular epithelioid } \\
\text { cell tumor (PEComa) }\end{array}$ & 58 & 20.7 & 15.5 & 0.0 & 1.7 & 1.7 & 1.7 & 5.2 & 0.0 \\
\hline Angiosarcoma & 292 & 20.2 & 16.4 & 0.3 & 1.7 & 0.3 & 1.0 & 3.1 & 0.3 \\
\hline $\begin{array}{l}\text { Endometrial stromal } \\
\text { sarcoma }\end{array}$ & 200 & 15 & 12.5 & 0.0 & 0.5 & 0.0 & 2.0 & 2.5 & 0.0 \\
\hline $\begin{array}{l}\text { Rhabdomyosarcoma } \\
\text { (Embryonal) }\end{array}$ & 96 & 14.6 & 10.4 & 2.1 & 1.0 & 0.0 & 1.0 & 2.1 & 0.0 \\
\hline $\begin{array}{l}\text { Rhabdomyosarcoma } \\
\text { (NOS) }\end{array}$ & 199 & 14.1 & 12.1 & 0.0 & 2.0 & 0.0 & 0.0 & 2.0 & 0.0 \\
\hline Hemangioendothelioma & 68 & 10.3 & 7.4 & 0.0 & 2.9 & 0.0 & 0.0 & 2.9 & 0.0 \\
\hline Uterus leiomyosarcoma & 678 & 10.2 & 9.3 & 0.3 & 0.1 & 0.1 & 0.1 & 0.4 & 0.1 \\
\hline $\begin{array}{l}\text { Inflammatory myofibro- } \\
\text { blastic tumor }\end{array}$ & 62 & 9.7 & 8.1 & 0.0 & 0.0 & 0.0 & 0.0 & 0.0 & 1.6 \\
\hline Uterus sarcoma (NOS) & 129 & 9.3 & 8.5 & 0.8 & 0.0 & 0.0 & 0.0 & 0.0 & 0.0 \\
\hline $\begin{array}{l}\text { Unknown primary leio- } \\
\text { myosarcoma }\end{array}$ & 206 & 8.7 & 7.8 & 0.0 & 0.0 & 0.0 & 1.0 & 1.0 & 0.0 \\
\hline $\begin{array}{l}\text { Rhabdomyosarcoma } \\
\text { (Alveolar) }\end{array}$ & 101 & 6.9 & 5.0 & 0.0 & 0.0 & 1.0 & 1.0 & 2.0 & 0.0 \\
\hline Leiomyosarcoma & 924 & 6.4 & 5.2 & 0.0 & 0.4 & 0.2 & 0.3 & 1.0 & 0.2 \\
\hline Solitary fibrous tumor & 149 & 6 & 6.0 & 0.0 & 0.0 & 0.0 & 0.0 & 0.0 & 0.0 \\
\hline Breast angiosarcoma & 70 & 5.7 & 5.7 & 0.0 & 0.0 & 0.0 & 0.0 & 0.0 & 0.0 \\
\hline Synovial sarcoma & 321 & 5 & 4.0 & 0.0 & 0.0 & 0.0 & 0.6 & 0.6 & 0.3 \\
\hline Liposarcoma & 805 & 5 & 4.5 & 0.1 & 0.1 & 0.1 & 0.1 & 0.4 & 0.0 \\
\hline $\begin{array}{l}\text { Desmoplastic small } \\
\text { round cell tumor }\end{array}$ & 113 & 4.4 & 4.4 & 0.0 & 0.0 & 0.0 & 0.0 & 0.0 & 0.0 \\
\hline $\begin{array}{l}\text { Alveolar soft part sar- } \\
\text { coma (ASPS) }\end{array}$ & 72 & 4.2 & 2.8 & 0.0 & 1.4 & 0.0 & 0.0 & 1.4 & 0.0 \\
\hline
\end{tabular}

a Children's Oncology Group (COG) protocol, failed to reproduce this finding [27]. Other work has also found an association between $C D K N 2 A$ deletion and poor prognosis in GIST [22].

CDKN2A (cyclin-dependent kinase inhibitor 2A) is a tumor suppressor gene that encodes two proteins: p16 and p14arf [28]. The p16 protein plays a functional role in cell cycle and senescence through the regulation of the cyclin-dependent kinase (CDK) $4 / 6$ and cyclin D complexes. p14arf activates TP53, the canonical tumor suppressor. CDKN2A loss of function is seen in a number of different cancer types, with the majority of cases being inactivation by homozygous deletions, followed by less common inactivating mutations and promoter hypermethylation. In our study, we found that loss of $C D K N 2 A$ had a significant correlation with worse prognosis in localized STS at our institution and validated this in an independent cohort of patients from TCGA. The histologic subtypes MPNST, MFS, and UPS had increased 


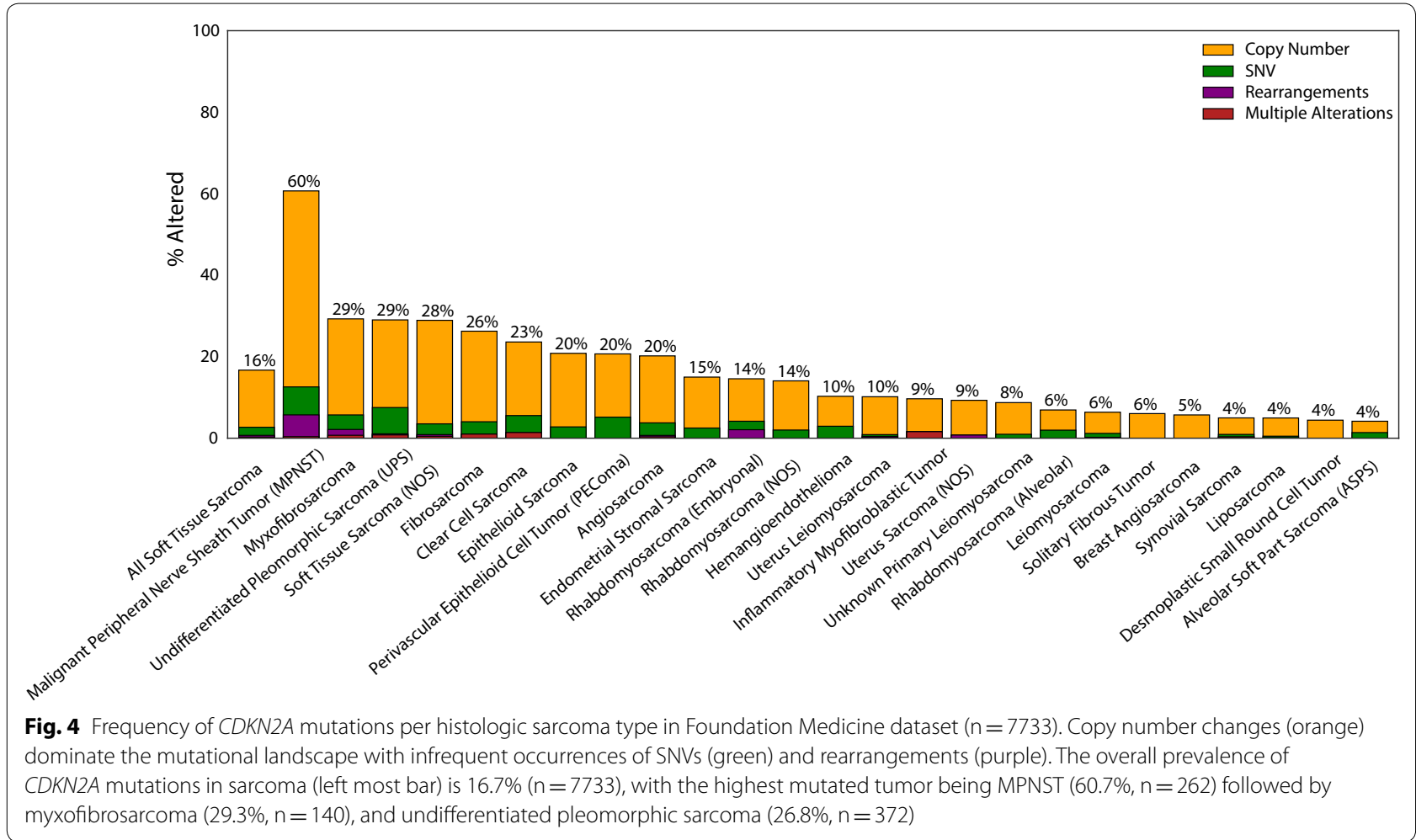

frequency of CDKN2A loss as compared to LMS and DDLPS.

This data can potentially help further risk stratify patients and inform which patients are at the highest risk of relapse and would warrant adjuvant chemotherapy. This would be especially helpful in STS as the concept of adjuvant therapy is controversial and there is no definitive consensus on whether it should be administered to all patients. Detection of genomic aberrations in $C D K N 2 A$ may also have therapeutic implications since a number of basket and umbrella clinical trials enroll patients with concordant loss of $C D K N 2 A$ and amplifications of $C D K 4, C D K 6, C C N D 1, C C N D 2$ and/or CCND3. We identified co-existing aberrations in these genes in a small subset $(1.5 \%)$ of sarcoma patients analyzed in the TCGA study and Memorial Sloan Kettering genomic studies (summarized in Additional file 1: Tables S1, S2). Sarcoma patients carrying these aberrations may be suitable candidates for clinical trials of CDK4 inhibitors.

There are some limitations to this study. First, STS encompass a wide range of natural history, with certain histologies behaving more aggressively, while others behave more indolently. In our single institutional cohort due to the wide variety of tissue types, we were unable to statistically control for tumor type due to anticipated loss of statistical power. However, in the larger TCGA dataset because of the more limited number of histologies, our statistical model accounted for tumor type, and CDKN2A was still a significant marker of poor prognosis. Furthermore, in our cohort, genetic sequencing was almost exclusively done on patients that developed advanced disease and thus as a byproduct, acted as a selector for aggressive biology (ex. $>90 \%$ of patients developed metastatic disease). Another limitation in our cohort is that sequencing was not done uniformly on the primary tumor at time of diagnosis, thus there could potentially be changes in the genome throughout the disease course that may not be captured by a single timepoint biopsy. In addition, there is the possibility of diverging clones that establish metastatic sites and are genetically distinct from the primary tumor. A potential solution to this is to use circulating tumor DNA (ctDNA) from peripheral blood, which theoretically includes DNA deposits from multiple tumor sites. We have recently shown the clinical utility of detection of SNVs, indels and copy number alterations in ctDNA of patients with LMS, and demonstrated that ctDNA analysis may capture the molecular intra-tumoral heterogeneity of LMS [29]. Another limitation is that in the Foundation One dataset, pathology is not centrally reviewed so the pathologic diagnosis is dependent on the diagnosis term sent in by the referring physician. This can lead to incorrect or outdated diagnoses, as manifested by the high prevalence of 
fibrosarcoma in the database. Finally, this is a single institutional experience and larger data sets will be beneficial in exploring this association further. In rare tumors, this can be challenging but can be achieved through the work of rare tumor consortiums.

\section{Conclusions}

In summary, we demonstrate the association between genomic aberrations affecting the CDKN2A gene and worse prognosis in two independent data sets of STS. We also establish the frequency of CDKN2A alterations across histologies in a large STS genomic database, although limited by lack of central review of pathology. Further research is needed into novel therapeutics to target the p16-CDK4-RB1 pathway, and whether additional adjuvant therapy upfront can improve outcomes in this subset of patients.

\section{Supplementary information}

Supplementary information accompanies this paper at https://doi. org/10.1186/s13569-019-0122-5.

Additional file 1. Additional tables and figures.

\section{Abbreviations}

BS: bone sarcoma; CDK: cyclin-dependent kinase; CDKN2A: cyclin-dependent kinase inhibitor 2A; ctDNA: circulating tumor DNA; COG: Children's Oncology Group; DDLPS: dedifferentiated liposarcoma; FNCLCC: Fédération Nationale des Centres de Lutte Contre Le Cancer; GIST: Gastrointestinal Stromal Tumor; HR: hazard ratio; IRB: Institutional Review Board; LMS: leiomyosarcoma; LPS: liposarcoma; MFS: myxofibrosarcoma; MPNST: Malignant Peripheral Nerve Sheath Tumor; NOS: not otherwise specified; OS: overall survival; SNV: single nucleotide variant; STS: soft tissue sarcoma; TCGA: The Cancer Genome Atlas; TTF: time to treatment failure; UPS: undifferentiated pleomorphic sarcoma; SS: synovial sarcoma.

\section{Acknowledgements}

This study was presented at the ASCO Annual meeting June 2, 2018.

\section{Authors' contributions}

NQB: designed the study, acquired and analyzed the data, drafted the manuscript, and supervised the study. JP: acquired and analyzed the data, and drafted the manuscript. SET: acquired and analyzed the data, and edited the manuscript. GF: helped in data acquisition. TH: analyzed the data and provided statistical support. MvdR: analyzed the data and edited the manuscript. KNG edited the manuscript. All authors read and approved the final manuscript.

\section{Funding}

No funding was received for this work.

\section{Availability of data and materials}

The datasets used and/or analysed during the current study are available from the corresponding author on reasonable request.

\section{Ethics approval and consent to participate}

This study was approved by the Stanford Institutional Review Board (IRB) of Stanford, CA.

\section{Consent for publication}

This manuscript does not contain data from any individual person.

\section{Competing interests}

SET is employed by and has patents with Foundation Medicine, Inc. and has equity in F. Hoffmann-La Roche Ltd. GF is employed by and has patents with Foundation Medicine, Inc. and has equity in F. Hoffmann-La Roche Ltd. KNG has consulting roles with Novartis, Daiichi Sankyo, Janssen, and Eli Lilly. NQB, $J P, T H, M v d R$ declare no competing interests.

\section{Author details}

${ }^{1}$ Department of Medicine (Oncology), Stanford University School of Medicine, 875 Blake Wilbur Drive, Stanford, CA 94305, USA. ${ }^{2}$ Department of Pathology, Stanford University School of Medicine, Stanford, CA, USA. ${ }^{3}$ Foundation Medicine, Cambridge, MA, USA. ${ }^{4}$ Department of Statistics, Stanford University, Stanford, CA, USA.

Received: 29 June 2019 Accepted: 28 August 2019

Published online: 11 September 2019

\section{References}

1. Callegaro D, et al. Development and external validation of two nomograms to predict overall survival and occurrence of distant metastases in adults after surgical resection of localised soft-tissue sarcomas of the extremities: a retrospective analysis. Lancet Oncol. 2016;17:671-80,

2. Sarcoma meta-analysis collaboration. Adjuvant chemotherapy for localised resectable soft-tissue sarcoma of adults: meta-analysis of individual data. Lancet. 1997;350(9092):1647-54.

3. Woll PJ, et al. Adjuvant chemotherapy with doxorubicin, ifosfamide, and lenograstim for resected soft-tissue sarcoma (EORTC 62931): a multicentre randomised controlled trial. Lancet Oncol. 2012;13:1045-54.

4. Le Cesne A, et al. Doxorubicin-based adjuvant chemotherapy in soft tissue sarcoma: pooled analysis of two STBSG-EORTC phase III clinical trials. Ann Oncol Off J Eur Soc Med Oncol. 2014;25:2425-32.

5. Pervaiz N, et al. A systematic meta-analysis of randomized controlled trials of adjuvant chemotherapy for localized resectable soft-tissue sarcoma. Cancer. 2008;113:573-81.

6. Bloomfield CD, Goldman A, Hossfeld D, de la Chapelle A. Clinical significance of chromosomal abnormalities in acute nonlymphoblastic leukemia. Cancer Genet Cytogenet. 1984;11:332-50.

7. Paik S, et al. A multigene assay to predict recurrence of tamoxifen-treated, node-negative breast cancer. N Engl J Med. 2004;351:2817-26.

8. Hegi ME, et al. Clinical trial substantiates the predictive value of O-6-methylguanine-DNA methyltransferase promoter methylation in glioblastoma patients treated with temozolomide. Clin Cancer Res. 2004;10:1871-4.

9. Poeta ML, et al. TP53 mutations and survival in squamous-cell carcinoma of the head and neck. N Engl J Med. 2007;357:2552-61.

10. Release FN. FDA announces approval, CMS proposes coverage of first breakthrough-designated test to detect extensive number of cancer biomarkers. 2017. https://www.fda.gov/NewsEvents/Newsroom/Press Announcements/ucm587273.htm. Accessed 4 May 2018.

11. He J, et al. Integrated genomic DNA/RNA profiling of hematologic malignancies in the clinical setting. Blood. 2016;127:3004-14.

12. Frampton $\mathrm{GM}$, et al. Development and validation of a clinical cancer genomic profiling test based on massively parallel DNA sequencing. Nat Biotechnol. 2013;31:1023-31.

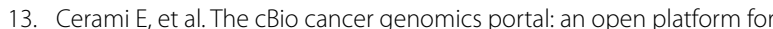
exploring multidimensional cancer genomics data: figure 1. Cancer Discov. 2012;2:401-4.

14. Sun JX, et al. A computational approach to distinguish somatic vs. germline origin of genomic alterations from deep sequencing of cancer specimens without a matched normal. PLOS Comput Biol. 2018;14:e1005965.

15. Team RC, others. R: A language and environment for statistical computing. 2013.

16. Therneau TM, Lumley. T. Package 'survival'. R Package version 2-41. 2017

17. Siegel RL, Miller KD, Jemal A. Cancer statistics, 2018. CA Cancer J Clin. 2018:68:7-30

18. Jouenne F, et al. Germline CDKN2A/P16INK4A mutations contribute to genetic determinism of sarcoma. J Med Genet. 2017;54:607-12. 
19. Nielsen GP, et al. Malignant transformation of neurofibromas in neurofibromatosis 1 is associated with CDKN2A/p16 inactivation. Am J Pathol. 1999;155:1879-84.

20. Sohier P, et al. Confirmation of mutation landscape of NF1-associated malignant peripheral nerve sheath tumors. Genes Chromosom Cancer. 2017;56:421-6.

21. Cancer Genome Atlas Research Network. Electronic address: elizabeth. demicco@sinaihealthsystem.ca A, et al. Comprehensive and integrated genomic characterization of adult soft tissue sarcomas. Cell. 2017; 171; 950-965.

22. Lagarde $P$, et al. Mitotic checkpoints and chromosome instability are strong predictors of clinical outcome in gastrointestinal stromal tumors. Clin Cancer Res. 2012;18:826-38.

23. Lagarde $\mathrm{P}$, et al. Chromosome instability accounts for reverse metastatic outcomes of pediatric and adult synovial sarcomas. J Clin Oncol. 2013;31:608-15.

24. Tsuchiya T, et al. Analysis of the p16INK4, p14ARF, p15, TP53, and MDM2 genes and their prognostic implications in osteosarcoma and Ewing sarcoma. Cancer Genet Cytogenet. 2000;120:91-8.

25. Brownhill SC, Taylor C, Burchill SA. Chromosome 9p21 gene copy number and prognostic significance of p16 in ESFT. Br J Cancer. 2007;96:1914-23.
26. Wei $G$, et al. Prognostic impact of INK4A deletion in Ewing sarcoma. Cancer. 2000;89:793-9.

27. Lerman DM, et al. Tumoral TP53 and/or CDKN2A alterations are not reliable prognostic biomarkers in patients with localized Ewing sarcoma: a report from the Children's Oncology Group. Pediatr Blood Cancer. 2015;62:759-65.

28. Collado M, Blasco MA, Serrano M. Cellular senescence in cancer and aging. Cell. 2007;130:223-33.

29. Przybyl J, et al. Combination approach for detecting different types of alterations in circulating tumor DNA in leiomyosarcoma. Clin Cancer Res. 2018;24:2688-99.

30. Gu Z, Eils R, Schlesner M. Complex heatmaps reveal patterns and correlations in multidimensional genomic data. Bioinformatics. 2016:32:2847-9.

\section{Publisher's Note}

Springer Nature remains neutral with regard to jurisdictional claims in published maps and institutional affiliations.
Ready to submit your research? Choose BMC and benefit from:

- fast, convenient online submission

- thorough peer review by experienced researchers in your field

- rapid publication on acceptance

- support for research data, including large and complex data types

- gold Open Access which fosters wider collaboration and increased citations

- maximum visibility for your research: over $100 \mathrm{M}$ website views per year

At $\mathrm{BMC}$, research is always in progress.

Learn more biomedcentral.com/submissions 